\title{
RESEÑA DE LIBRO: Ospina Mejía, Oswaldo y Alvarado-Peña, Lisandro (eds). (2020). Gestión organizacional, sustentabilidad, innovación y emprendimiento en América Latina. Universidad Sergio Arboleda.
}

\author{
Gumaro Álvarez Vizcarra* \\ https://orcid.org/0000-0003-4809-1012
}

\begin{abstract}
Como citar: Álvarez Vizcarra, Gumaro. (2021). Reseña de libro: Ospina Mejía, Oswaldo y AlvaradoPeña, Lisandro (eds). (2020). Gestión organizacional, sustentabilidad, innovación y emprendimiento en América Latina. Universidad Sergio Arboleda. Telos: revista de Estudios Interdisciplinarios en Ciencias Sociales, 23 (2), Venezuela. (Pp. 504-508).

DOI: www.doi.org/10.36390/telos232.18
\end{abstract}

En América Latina las asimetrías son una de las características, historias, tradiciones y estructuras sociales compartidas, las disparidades entre países y al interior de ellos, azotan los rostros de sus habitantes, la falta de oportunidades para unos, el desempleo y la profunda desigualdad en la distribución del ingreso, son algunas de las más evidentes realidades. Los países latinoamericanos y sus procesos pujantes por abordar el desarrollo exigen de sus ciudadanos, esfuerzos extraordinarios para promover iniciativas que transformen el ecosistema empresarial, político, social, académico, como bastiones indispensables para transitar a entornos sustentables e innovadores, en los cuales la capacidad para crear unidades de negocio se vea fortalecida y promueva el crecimiento, la competitividad y una sociedad justa e incluyente.

En este contexto, aparece en el escenario académico-científico, el libro "Gestión Organizacional, Sustentabilidad, Innovación y Emprendimiento en América Latina" una compilación cuyos coordinadores se dieron a la tarea de seleccionar excelentes trabajos, bajo un proceso riguroso de revisión por pares. La edición e impresión fue realizada por la Editorial de la Universidad Sergio Arboleda, y la Red Académica Internacional de Estudios Organizacionales en América Latina, el Caribe e Iberoamérica -REOALCel-, en Bogotá, Colombia, en el año 2020.

La preocupación de los coordinadores por producir una obra que tome en cuenta la problemática que vive la región latina, la reafirman al procurar incluir trabajos en un ambiente interdisciplinario y transdisciplinario, de tal manera que las diversas disciplinas de la ciencia se entremezclen y se asegure su conexión transfronteriza, garantizando el desarrollo de las temáticas que los problemas presentes en la region latinoamericana demandan, con el objetivo claro de contribuir en el logro de mejores indicadores macroeconómicos y bienestar social. Desde esta perspectiva, con una congruencia total, se define la estructura del libro, adicional a

* Tecnológico de Monterrey, Sinaloa. México. Correo electrónico: galvarez@tec.mx 
la introducción, y al prólogo, la obra está compuesta por tres grandes apartados, en los cuales se distribuyeron 12 artículos, tal como aparecen a continuación.

1. Gestión organizacional (3)

2. Estudios socioeconómicos y sostenibilidad (6)

3. Emprendimiento e innovación (3)

Evidentemente, existe una congruencia entre el título de la obra y la segmentación utilizada, situación que pone en evidencia, el cuidado que tuvieron los coordinadores, para darle una estructura concatenada, haciendo posible llevar al lector paso a paso por los contenidos ofrecidos.

La gestión organizacional, es un constructo con demasiada historia, así como complejidad en su tratamiento y asimilación, su evolución desde los principios de las teorías clásicas, con los primeros planteamientos para medir el desempeño realizados por Tylor, la distribución de funciones de Henry Fayol; los controles de la teoría burocrática de Weber, pasando por la de Follet, Maslow, Barnard, entre otros; hasta las consideradas teorías modernas, de Hannan y Freeman, Di Maggio, Williamson y Kaufmann con su teoría de los sistemas complejos.

El debate sobre la administración de las organizaciones ha sido constante e intenso, existe una cantidad abrumadora de teorías y corrientes preocupadas por explicar lo que sucede en el campo de la gestión organizacional, sin embargo, el carácter dinámico de las organizaciones cotidianamente muestra signos evolutivos que fecundan nuevas condiciones de análisis. En esta ocasión, si bien no se desarrolla una nueva teoría, el texto que hoy nos ocupa, es una obra que contribuye directamente al fortalecimiento de la administración organizacional, proporcionando experiencias empíricas, teóricas y académicas, enmarcadas en el proceso de reflexión para diseñar políticas y objetivos de desempeño, orientadas al desarrollo económico de la región latinoamericana y del Caribe, promoviendo mejores oportunidades de vida, igualdad económica, inclusión financiera, y aceptación social.

El texto, en su primer apartado pone sobre la mesa de la discusión tres interesantes capítulos. En el primero, los autores realizan un análisis profundo del impacto de la estructura de mercado, en la competitividad empresarial. La presencia o ausencia de competencia, define el tipo de mercado en el que una empresa se desarrolla, un mercado eficiente, presupone la existencia de la libre y total competencia, no obstante, la aparición de imperfecciones, van encaminando a las organizaciones a mercados con ausencia de competencia, situándolo en mercados imperfectos, como el caso que en este capítulo se aborda.

Desde los planteamientos de Adam Smith, hasta los contemporáneos, el debate va más allá del equilibrio entre oferta y demanda, es decir, las perturbaciones del mercado, trastocan los excedentes de los agentes económicos, por tanto promueven la transferencia y con ello la ineficiencia, en este sentido, sucintamente los autores del primer capítulo, estudian el proceso de "cartelización" como un fenómeno que genera el desarrollo de un mercado poco competido, con efectos negativos para las empresas carentes de poder de mercado, consecuentemente afecta la competitividad.

Por lo general, cuando en los proceso administrativos se presenta un cambio disruptivo, se enfrenta a una disyuntiva entre la resistencia de las inercias establecidas por el tiempo, y las exigencias de las nuevas formas de operar, este es el caso que se expone en el segundo capítulo; migrar de un modelo de negocio enraizado en las tradiciones tanto 
RESEÑA DE LIBRO: Ospina Mejía, Oswaldo y Alvarado-Peña, Lisandro (eds). (2020). Gestión organizacional, sustentabilidad, innovación y emprendimiento en América Latina.

operativas como sociales comunitarias, a uno distinguido por el uso de la tecnología y las relaciones impersonales, en este texto, la técnica descriptiva que utilizan los autores, invitan al lector a continuar explorando las ideas planteadas de una manera mesurada, casi artística.

Entre anécdotas, datos históricos, se describe una realidad que caracteriza a la mayoría de los pueblos de América Latina y el Caribe, la controversia entre la tradición y la modernidad, hasta donde hay que sostener una y con qué intensidad promover la otra, este debate lo muestran de manera magistral, pues describir los desafíos de una organización en el proceso de sustitución del modelo de negocio, en el cual hay que romper inercias y tradiciones que trastocan la vida misma de los grupos de interés, los autores desmenuzan concienzudamente el caso de rio Magdalena, afluente de gran trascendencia para economía colombiana. En el capítulo se pone en evidencia la necesidad de ser capaces de mezclar paulatinamente la experiencia empírica tradicional con los procesos modernos, para efectos de garantizar una transición exitosa, evitando la ruptura con los colaboradores, mejorando el clima laboral y las condiciones de vida.

El segundo apartado del texto que nos ocupa está constituido por seis capítulos, exponiendo un escenario heterogéneo, la discusión se plantea desde diversas perspectivas y sobre tópicos de gran envergadura para el desarrollo de la región. Además de ser el apartado más nutrido, los lectores encontraran una serie de planteamientos que los transportará a realidades muy diversas, al rescatar elementos de estudio de países como Chile, Colombia, Uruguay y México, esta diversidad, facilita entender las asimetrías internas de cada país, pero además las existente entre ellos. Los capítulos cuatro, cinco y seis, se sumergen en el análisis de problemáticas sociales, que han permeado por años en las sociedades de estos países y que se han convertido en pesadas anclas que obstaculizan el tránsito hacia el desarrollo.

En el capítulo cuatro, se realiza un estudio profundo sobre el desempleo juvenil en Chile, los autores realizan una descripción precisa del proceso metodológico, que les permitió desarrollar modelos econométricos a partir de insumos obtenidos de la encuesta nacional, encontrando evidencias muy interesantes sobre la estructura poblacional y los determinantes sociológicos del desempleo en un contexto poco usual de un sector de la población para el cual debería existir mayores oportunidades, en este capitulo se entretejen las estructuras y se muestran las diferencias y marginaciones sociales, un escenario fácilmente identificable en economías de la región.

Los capítulos cinco y seis, muestran una realidad Colombiana desde dos perspectivas, primero, se expone la ausencia de una educación financiera, los autores desnudan perfectamente este tópico, al mostrar la existencias de fallas profundas en el sistema educativo, y en el sistema financiero colombiano, la confluencia de ambos tiene un impacto en el agravamiento de la exclusión financiera, y por tanto el ensanchamiento de la brecha entre la población bancarizada, la no bancarizada, y el sesgo en las oportunidades de desarrollo. aunque en el debate se argumenta que la bancarización debe darse por demanda, no es posible desarrollar una demanda, si la infraestructura física y territorial de los bancos no está lo suficientemente desarrollada para llegar a los usuarios.

El segundo caso, es una exposición magistral de un problema que ha trastocado la profundidad de la estructura social colombiana, "el conflicto armado", como toda confrontación, sus costos son onerosos y dolorosos, lo primero, porque son grandes las cantidades de dinero 
puestas para su atención; los segundos, porque resquebrajan el tejido social, causando dolor, muerte, desestabilidad emocional y desequilibrio social.

Los autores, tienen una agudeza y claridad para encontrar las áreas sensibles de este fenómeno, concluyendo que los esfuerzos realizados para atender la prestación humanitaria, como una estrategia para resarcir el daño, han sido buenos, pero, categóricamente insuficientes, pues dada la complejidad del problema se requieren esfuerzos mayores que garanticen la imparcialidad en la llegada de los beneficios en muchas áreas de la desigualdad social.

Los dos capítulos faltantes para cerrar el segundo apartado del texto exponen dos estudios diferentes, pero interesantes, en uno, se habla de las ventajas de atender la condición de un grupo de empresas promoviendo el acercamiento a los indicadores que sugiere la norma ISO 26000, tanto para el tema social, como para la inversión para el desarrollo. En el otro, los autores proponen la implementación de un modelo de desarrollo turístico sustentable, considerando la existencia de condiciones favorables en los aspectos geográficos, demográficos, de flora y fauna silvestre, enfatizando en la virtud de promover el desarrollo social, contribuyendo en la disminución de la marginación y la pobreza.

El tercer y último apartado que conforma la estructura del texto está dedicado al emprendimiento, emprendimiento social, e innovación. Si se habla de innovación y emprendimiento, las condiciones de las economías modernas y su índice de mutación deben convertirse en los principales motivadores para iniciar por el camino de la innovación y el emprendimiento. Innovar es moverse a mayor velocidad en un espacio entrópico, es decir, introducirse en una espiral ascendente sustituyendo estados de equilibrio, para iniciar otras formas, conceptos, ideas, y proyectos. Evidentemente, emprender e innovar son preceptos con una alta correlación.

El emprendimiento es la puerta para la generación de nuevas oportunidades de crecimiento personal, profesional, empresarial y social, significa dar el paso inicial para la cristalización de un proyecto. Este proceso de construcción implica introducirse en la esfera de la innovación, pues emprender es modificar procesos, es desechar estructuras, es crear espacios, es fortalecer conceptos, es irrumpir en lo desconocido, y esto, implícitamente, es caminar senderos de la innovación en un entorno incierto. Los autores enfatizan en los criterios para definir el emprendimiento social, así como sus variables determinantes, en un escenario segmentado cuyas partes responden a condiciones sociales diferentes, con perfecta claridad se establece la condición transformadora del emprendimiento en el desarrollo de las regiones, pues de él se deriva la generación de nuevos proyectos, la creación de empleos y con ello el fortalecimiento del bienestar y la riqueza social.

Las características y la complejidad de los fenómenos que determinan el desempeño de las economías de Latinoamérica dificultan la comprensión, el análisis y la definición de las variables que en ella inciden. Por tanto, ante la diversidad y las asimetrías, se requieren múltiples esfuerzos colegiados, en el cual participen, los gobiernos, los empresarios, la población y las universidades, siendo estas últimas, las responsables de asumir el liderazgo en la generación de conocimientos, que vengan a promover economías con igualdad, inclusión y sustentabilidad.

En este escenario, adquiere importancia la propuesta académica de la presente obra, esfuerzos como este son importantes como detonantes del cambio desde la perspectiva social, 
RESEÑA DE LIBRO: Ospina Mejía, Oswaldo y Alvarado-Peña, Lisandro (eds). (2020). Gestión organizacional, sustentabilidad, innovación y emprendimiento en América Latina.

empresarial y gubernamental de los pueblos de América Latina y el Caribe, para promover el crecimiento, mejorando la calidad de vida de sus habitantes. Hoy en día, en un contexto altamente incierto, los esfuerzos colegiados representan un acierto en la mejora continua de la gestión organizacional.

Puede descargarlo en: https://repository.usergioarboleda.edu.co/handle/11232/1430

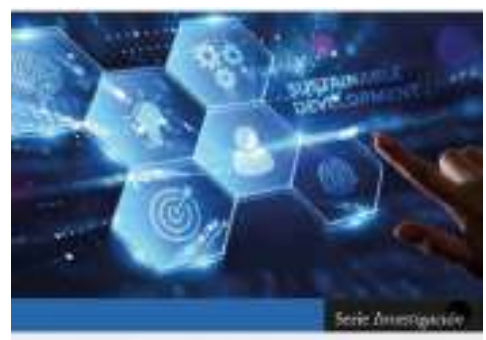

GESTIÓN ORGANIZACIONAL.

SUSTENTABILIDAD, INNONACTÓN

YEMPRENDIMIENTO EN

AMERICALATINA

Ormbitheiv ving

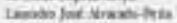

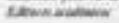

Q 